\title{
Induktion selbstschädigender Handlungen besonders unter hohen Dosierungen
}

Fragestellung: Die Studie erfasste das Risiko selbstschädigenden Verhaltens unter Antidepressiva in Abhängigkeit von der Dosierung und Altersgruppe.

Hintergrund: Metaanalysen von randomisierten, kontrollierten Studien konnten zeigen, dass suizidales Verhalten bei Kindern, Jugendlichen und jungen Erwachsenen etwa doppelt so häufig unter Antidepressiva im Vergleich zu Placebo auftritt. Bei Erwachsenen über 24 Jahren wurde dagegen kein erhöhtes Risiko ermittelt. Die Bedeutung der Dosierung und der Altersgruppe für dieses Risiko wurde bisher nicht systematisch untersucht.

Patienten und Methodik: Die prospektive Kohortenstudie untersuchte 162.625 Personen der US-Bevölkerung mit Depression im Alter zwischen 10 und 64 Jahren, bei denen eine Behandlung mit selektiven Serotonin-Wiederaufnahmehemmern (SSRI) neu initiiert wurde. Dabei wurden die Gruppen mit „modaler“ Dosis (= die am häufigsten gegebene Dosis) mit den Gruppen mit höheren Dosen verglichen. Haupt-Outcome-Kriterium waren die nach ICD-9 erfassten selbstschädigenden Handlungen der Kategorien E950.x-E958.x, also

Miller M, Swanson SA, Azrael D et al. Antidepressant dose, age, and the risk of deliberate selfharm. JAMA Intern Med 2014; 174: 899-909 alle selbstschädigenden und suizidalen Handlungen. Die modalen Dosen lagen für Citalopram bei $20 \mathrm{mg}$, für Sertralin bei $50 \mathrm{mg}$ und für Fluoxetin bei $20 \mathrm{mg}$.
Ergebnisse: In der Gruppe der 10- bis 24-Jährigen war die Rate selbstschädigender Handlungen annähernd doppelt so hoch bei höherer als bei modaler Dosis. Dies entsprach einem zusätzlichem Ereignis pro 150 behandelter Patienten. In der Altersgruppe der 25- bis 64-Jährigen war das absolute Risiko für suizidales Verhalten deutlich niedriger und es gab keine effektiven Risikounterschiede zwischen den Gruppen mit der modalen Dosis und der höheren als der modalen Dosis. Die meisten Ereignisse traten in den ersten drei Monaten nach Beginn der antidepressiven Behandlung auf. Der Risikounterschied zwischen hoher und modaler Dosis zeigte sich aber auch, wenn ein 1-Jahres-Zeitraum nach Beginn der Behandlung betrachtet wurde. Der Unterschied zeigte sich auch nach Matching entsprechend vorbestehenden suizidalen Tendenzen (propensity score matching).

Schlussfolgerungen: Depressive Patienten, die jünger sind als 24 Jahre, haben ein höheres Risiko für selbstschädigende Handlungen, wenn höhere als übliche Dosierungen von SSRI verordnet werden. Vor dem Hintergrund, dass kürzliche Metaanalysen auch gezeigt haben, dass die Wirksamkeit von Antidepressiva in diesen Altersgruppen nur mäßig gut ist und zwischen Antidepressivadosis und Wirksamkeit oft kein Zusammenhang ermittelbar ist, sprechen die Ergebnisse dafür, einerseits generell höhere Dosen für Antidepressiva bei diesen Altersgruppen zu vermeiden und bei Antidepressivabehandlung ein engmaschiges Monitoring, insbesondere in den ersten drei Monaten durchzuführen.

\section{- Kommentar von Ulrich Voderholzer, Prien am Chiemsee}

\section{Bei jugendlichen Depressiven mehr auf Psychotherapie setzen}

Die Untersuchungen zeigen die Dosisabhängigkeit des Risikos der Induktion suizidaler Verhaltensweisen bei depressiven Patienten unter 24 Jahren und mahnen zur Vorsicht bei der Anwendung dieser Substanzen. Insbesondere die Verhaltensaktivierung, also Induktion innerer Unruhe und Antriebssteigerung vor Beginn einer Stimmungsaufhellung scheinen mit diesem Risiko verbunden zu sein. Einschränkend muss natürlich darauf hingewiesen werden, dass bezüglich des Antidepressivaeinsatzes bei Kindern, Jugendlichen und jungen Erwachsenen immer eine Risikoabwägung erfolgen muss, da dem inzwischen belegten Risiko der Induktion selbstschädigenden Verhaltens auch das Risiko der Suizidalität bei fehlender Besserung im Rahmen schwerer Depressionen gegenübersteht. Ob das Risiko selbstschädigenden Verhaltens durch Antidepressivagabe im Vergleich zu einer Kontrollgruppe ohne Antidepressiva erhöht wird, kann durch die vorliegende Erhebung nicht beantwortet werden, da keine entsprechende Kontrollgruppe eingeschlossen war, ist aber bereits durch frühere
RCT mit Placebogruppen im Sinne eines erhöhten Risikos unter Antidepressiva geklärt worden. Wenn also überhaupt Antidepressiva in diesen Altersgruppen eingesetzt werden, sollten hohe Dosen vermieden werden. Bei Depressionen im Kindes-, Jugend- und jungen Erwachsenenalter sollte auch stärker als bisher Psychotherapie zum Einsatz kommen. Leider scheitert dies in der Praxis oft daran, dass Therapieplätze nicht rasch ge-

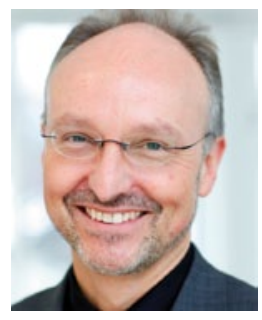

Prof. Dr. med. Ulrich Voderholzer, Prien am Chiemsee

Ärztlicher Direktor

Schön Klinik Roseneck, Psychosomatik

E-Mail: uvoderholzer@schoen-kliniken.de 